\author{
D. Downes \\ Institut de Radioastronomie Millimétrique \\ Voie 10, Domaine Universitaire \\ 38406 St. Martin d'Hères \\ France
}

\begin{abstract}
The massive $O B$ stars in our Galaxy form predominantly in the warm giant molecular clouds which constitute the spiral arms. The clouds are subject to a variety of mechanisms which retard or prevent further contraction, but are nevertheless able to form stable "cores". In the regime of subsonic internal motions, the cores may be regarded as potential protostars. The formation of massive cores, which then form massive stars, may initially be determined by the statistics of fragmentation, but may then be a feedback process, once underway, due to the steep increase of the minimum Jeans' mass with increasing temperature of the surroundings. This concept is the basis for the model of bi-modal star formation, and its implications for the initial mass function and the distribution of massive stars and metallicity gradients in the Galaxy.
\end{abstract}

\title{
1. THE CURRENT MODEL
}

1.1 Molecular clouds: large, warm ones and small cold ones

According to current ideas, atomic hydrogen clouds in the interstellar medium contract to form molecular clouds. Further condensations in molecular clouds form stable "cores", which contract under a suitable change in the external conditions to form protostars with flatted disks and outflows which eventually clear away sufficient material so that we can then observe the object, now nuclear-burning, as a pre-main sequence star. In this process, the critical number densities of hydrogen are thought to be as follows:

$10^{2} \mathrm{~cm}^{-3}$ : Formation of molecular clouds from the atomic gas;

$10^{4} \mathrm{~cm}^{-3}$. contraction along galactic magnetic field lines.

$10^{4} \mathrm{~cm}^{-3}$ : Radiative coupling of gas and dust, leading to core-

$>10^{6} \mathrm{~cm}^{-3}$. envelope structures and hydrostatic equilibrium in cores.

$>10^{6} \mathrm{~cm}^{-3}$ : some ion drift relative to neutrals; allows further

$>1011-3$ contraction (see models by Nakano 1984).

rapid loss of magnetic field (Nakano and Umebayashi 1986).

In protostellar disks, this process may drive bi-polar outflows (Pudritz and Norman 1983, Uchida and Shibata 1985). 93

M. Peimbert and J. Jugaku (eds.), Star Forming Regions, 93-109.

(C) 1987 by the IAU. 
The process appears to have two branches, as follows:

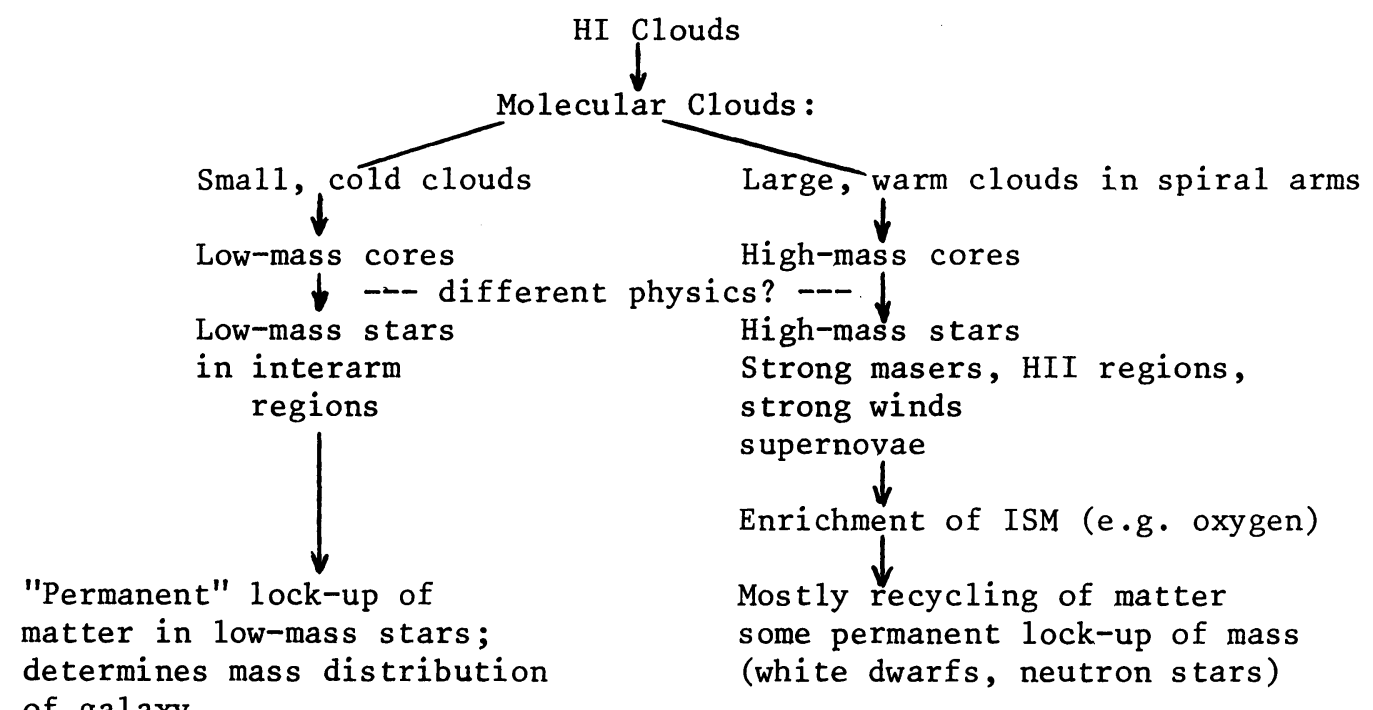

Fig. 1 Schema for low and high-mass modes of star formation.

The left branch in Fig. 1 corresponds, crudely speaking, to the process in interarm regions, and that in the right branch to spiral arm regions. There are, again roughly speaking, two types of molecular clouds: large warm ones and small cold ones (Table 1 ). The small cold clouds are found throughout the galactic disk, while the giant molecular clouds (GMC's), and supercloud complexes of GMC's, constitute the spiral arms of our Galaxy.

The GMC's do not form simply by random coagulation of small clouds in the general interstellar medium, but are presumably built up from the smaller clouds in the spiral density wave. The massive stars form predominantly in the warm GMC's, while mainly low-mass stars form in small, cold clouds. The spiral density-wave shocks do not sufficiently compress the gas to form stars directly, but the pressure is sufficient to induce the gas to form supercloud complexes (e.g. Jura 1976, Elmegreen this symposium). Alternatively, the GMC's may form in the spiral arms due to increased density of clouds at crossing points of orbits generated in response to bar forcing (Combes and Gerin 1985). In either case, the rate of formation of massive stars is indirectly increased relative to that in the interarm region, probably in proportion to the amount of gas flowing through the spiral arms.

This division of clouds into two types, the large warm ones in spiral arms, and small cold ones uniformly distributed in the galactic disk, is supported by recent analyses of CO surveys (Robinson et al. 1986, Sanders et a1. 1986, Stark and Bally 1987, Myers et al. 1986, Cohen et al. 1986), and their comparison with HII regions seen on the Bonn and Parkes radio continuum and recombination-line surveys, as well as with FIR surveys. The result of these comparisons is that all HII 
Table 1. Characteristics of Molecular Clouds

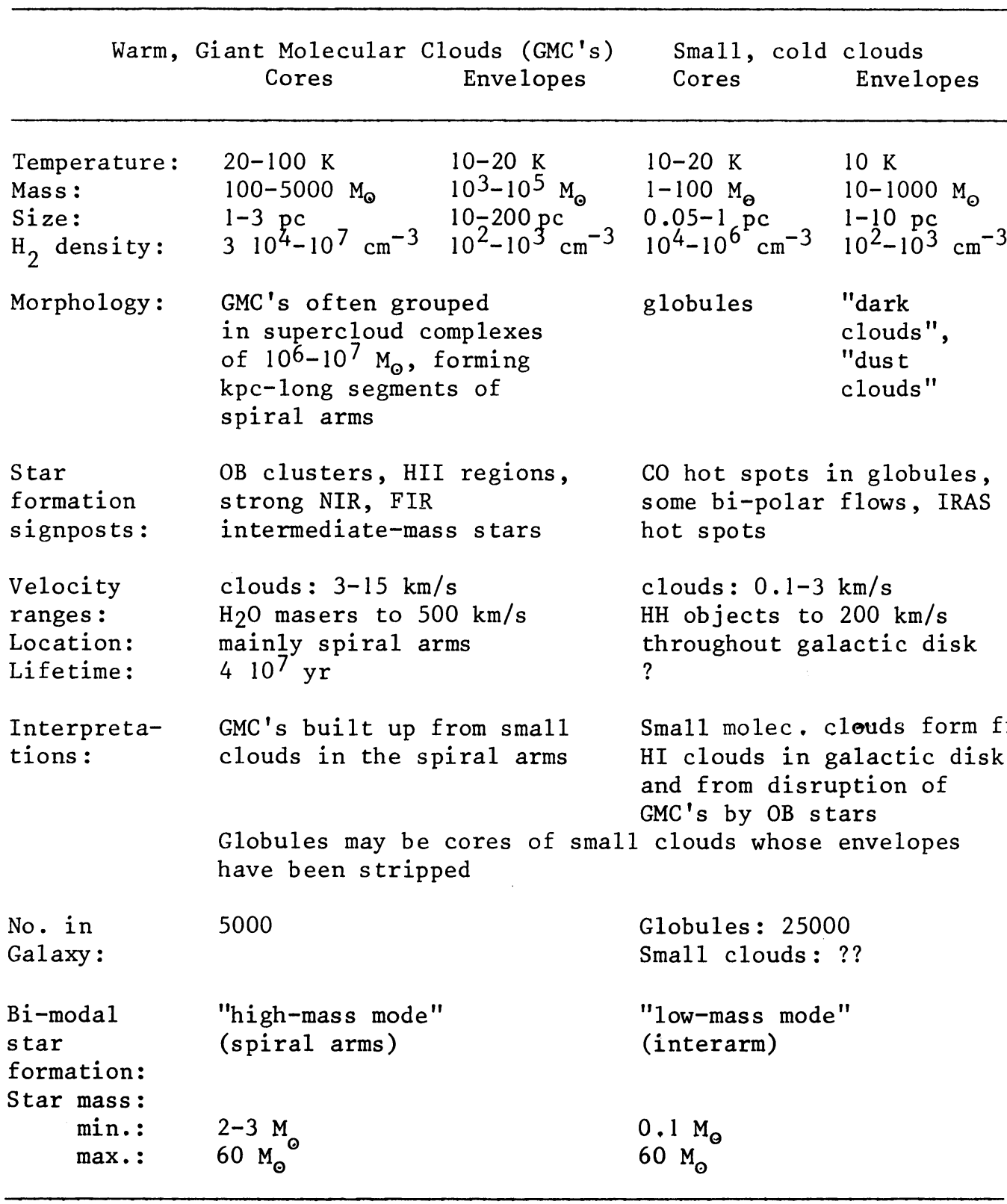

Refs: Cloud properties: Turner (1984), Evans (1985), Myers (1985)

Interpretations: No. in Galaxy: Solomon and Sanders (19.85)

Bi-modal star formation: Güsten and Mezger (1983);

Variation of star mass with metallicity: Kahn (1974)

Globules: Leung (1985); lifetimes: Bash et al. (1977)

Formation of GMC's: Combes and Gérin (1985) 
regions in the inner Galaxy with peak fluxes > $1 \mathrm{Jy}$ in a $2.6^{\prime}$ beam at $5 \mathrm{GHz}$ are associated with molecular clouds more massive than $10^{5} \mathrm{M}_{\odot}$. For a typical distance of $6 \mathrm{kpc}$, this flux limit corresponds to HII regions with radio luminosities equal to or greater than that of the Orion Nebula, so in the inner Galaxy, massive stars ionizing these large HII regions all seem to be formed in GMC's (For distances greater than a few kpc, weaker HII regions, such as NGC 2024 or Mon R2, would be below the $1-J y$ flux limit of the radio-line surveys).

\subsection{Stability of Molecular Clouds}

Once the clouds have formed, further contraction is slowed or stopped by a variety of mechanisms. Those discussed in the literature have been:

1) supersonic turbulence

2) rotation

3) winds (both protostar outflows and main-sequence winds)

4) magnetic fields

5) "gravito-turbulence", or "star cloud turbulence"

1) Supersonic turbulence: Although suggested by the "supersonic" linewidths of molecular lines observed in many clouds, it is generally agreed that supersonic turbulence, if unreplenished, is ineffective in supporting the clouds, as it will decay on a time scale comparable with the gravitational free-fall time.

2) Rotation is equally unlikely for the majority of clouds, as equilibrium rotation, balancing gravity, is not observed in most clouds, possibly because of magnetic braking of the cloud rotation.

3) Winds: To explain the internal kinetic energy of giant molecular clouds in the Galaxy, which is of the order of magnitude 1000 clouds $\mathrm{x}(3 \mathrm{~km} / \mathrm{s})^{2} \times 10^{5} \mathrm{M}_{\odot}=410^{50}$ erg, lasting $10^{7} \mathrm{yr}$, the likely cloud lifetime, one requires, for conversation of momentum, (no. of flows $x(M v) / f l o w=($ No. of clouds) $x(M \Delta v) / c l o u d$ or, Mv per flow $=310^{39}$ erg $/(\mathrm{cm} / \mathrm{s})=10 \mathrm{M}_{\Theta} \mathrm{km} / \mathrm{s}$ per flow, or an energy of 1045 erg per flow, for a flow velocity of $10 \mathrm{~km} / \mathrm{s}$ or, $\quad 1046$ erg per flow, for a flow velocity of $100 \mathrm{~km} / \mathrm{s}$ (Silk, this symposium; see similar calculation for Main-Sequence type winds by Kahn, this symposium).

While these values are certainly in the range of the larger molecular flows, such as Orion $510^{47} \mathrm{erg}$, or Mon R2, $11047 \mathrm{erg}$, it may not be possible to provide this average energy per flow with the lower mass outflows (e.g. T Tau 41043 erg; but see Beckwith et al. 1983). 4) Magnetic fields: Does cloud contraction take place in spite of magnetic fields? Arguments found in recent reviews are as follows: 


$$
\text { Pro }
$$

1) The magnetic field energy has the same $1 / R$ dependence as the gravitational field energy, so once contraction starts, the magnetic field can't stop it.

2) The collapse can proceed along the field lines.

3) Plasma drift (relative to neutrals) permits collapse.

4) Zeeman effect measurements indicate quite low fields in some clouds (e.g. Cas A, v=0, Schwartz et al. 1986, Heiles and Stevens 1986)

5) Clouds are supported by turbulence, not magnetic fields.

\section{Contra}

This is only true in the unrealistic case of smooth, spherical symmetry. In fact, as soon as tension develops and the field lines become tangled, it is possible for the magnetic energy to dominate the dynamics.

Probably only true at densities $<100 \mathrm{~cm}^{-3}$. Otherwise impossible, as it would require collimation of the collapse over 14 orders of magnitude of scale size.

only for densities $>10^{6} \mathrm{~cm}^{-3}$

Magnetic energy can dominate for cloud masses

$M>M_{C r} \sim 10^{3} M_{\odot} \frac{B}{30 \mu G}\left(\frac{R}{2 p c}\right)^{2}$
$M_{C r}=10^{5} M_{\odot}$ for $R=20 p c, B=30 \mu G$

(Mouschovias 1976, Shu, this symposium).

Interstellar polarization indicates large-scale magnetic field patterns, ruling out small-scale turbulence as cloud support.

An interesting development is that torsional Alfven waves can spin up cloud fragments as well as brake them (Mouschovias and Morton 1985). Thus even at the more condensed phases of molecular clouds, magnetic fields can hinder further contraction as well as aiding it.

Current observational data on magnetic fields (Fig. 2, Troland and Heiles 1986) suggest magnetic field strengths of $10-20 \mu$ Gauss, independent of cloud density, over the range 0.1 to $100 \mathrm{~cm}^{-3}$, consistent perhaps with cloud contraction parallel to the local magnetic field lines for this range of cloud densities. For the regime $10^{2}-10^{4} \mathrm{~cm}^{-3}$, the field appears to increase with density, consistent with predictions (Mouschovias 1976), with large variations from cloud to cloud. Some low-density clouds with supersonic turbulence, and which are not gravitationally bound, appear to have very high fields (condensations seen toward Cas A), while other, "dense" clouds have very low fields (local molecular clouds seen toward Cas A, Heiles and Stevens 1986). 


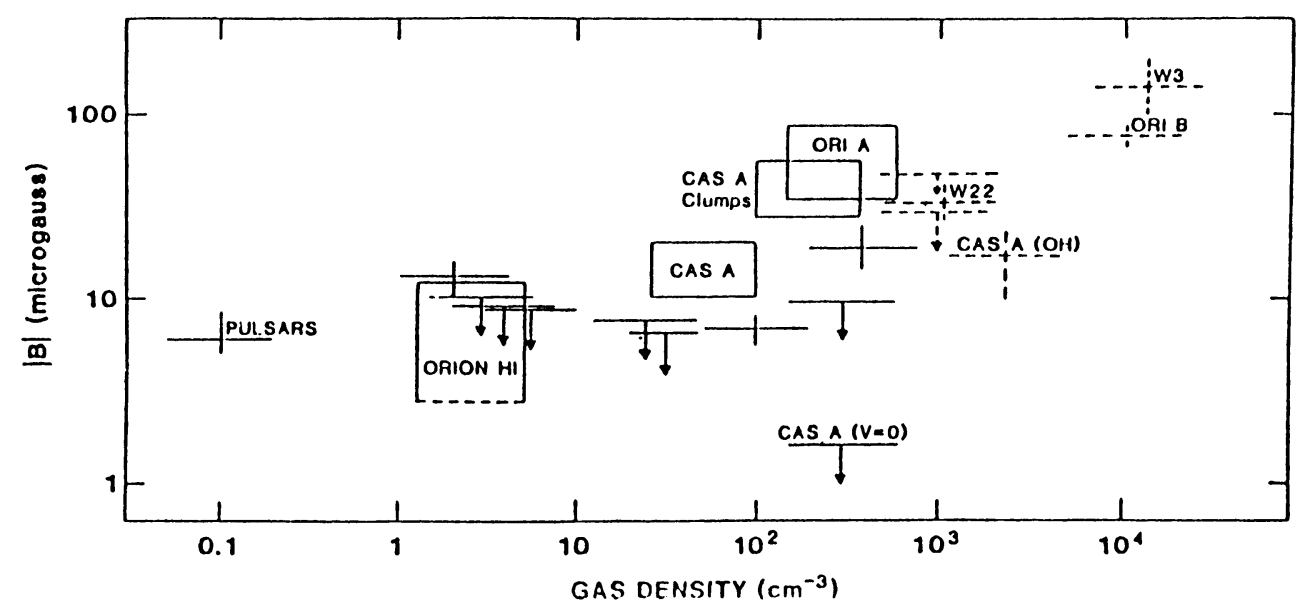

Fig. 2 Observed magnetic field strengths in intersteılar clouds, as a function of estimated number density of hydrogen (Troland and Heiles 1986).

5) "Gravito-turbulence" or "star cloud turbulence" has been discussed by Fleck $(1980,1981,1983)$, Henrickson and Turner (1984) and Larson (1984, 1985). The idea is that an ensemble of clouds with a gravitational correlation length, $\ell$, can "emit" a gravity tide to a larger-scale ensemble with correlation length $\mathrm{L}$. The smaller scale ensemble spins down, the larger-scale ensemble spins up. Simulation of three dimensional collapses (Larson 1978) show development of trailing spiral features which transfer angular momentum outward, reminiscent of calculations of galaxy-galaxy encounters. If this process transfers constant angular momentum per unit time per unit volume, $\rho_{\ell} v^{2}$ $=\rho_{L}\left(v_{L}\right)^{2}$, then the expectation is that the clouds will have $\Delta \mathrm{V} \sim \mathrm{L}^{0.5}$ and $\mathrm{M}$ (cloud) $\sim \Delta \mathrm{V}^{4}$

The observational results (Fig. 3), for a sample of cloud condensations with no strong internal heat sources, give

$$
\sigma(\mathrm{km} / \mathrm{s})=0.8 \mathrm{R}_{\mathrm{pc}} 0.5
$$

and

$$
\Delta \mathrm{V}=0.37\left(\mathrm{M} / \mathrm{M}_{\odot}\right)^{1 / 4}
$$

where $\sigma$ is the three-dimensional velocity dispersion. Substituting the velocity of sound, $v_{s}(\mathrm{~km} / \mathrm{s})=0.2(\mathrm{~T} / 10 \mathrm{~K}) 172$, one has for the minimum Jeans' Mass

$$
\mathrm{M} / \mathrm{M}_{\odot}=0.26(\mathrm{~T} / 10 \mathrm{~K})^{2} \quad(\text { Turner 1984) }
$$



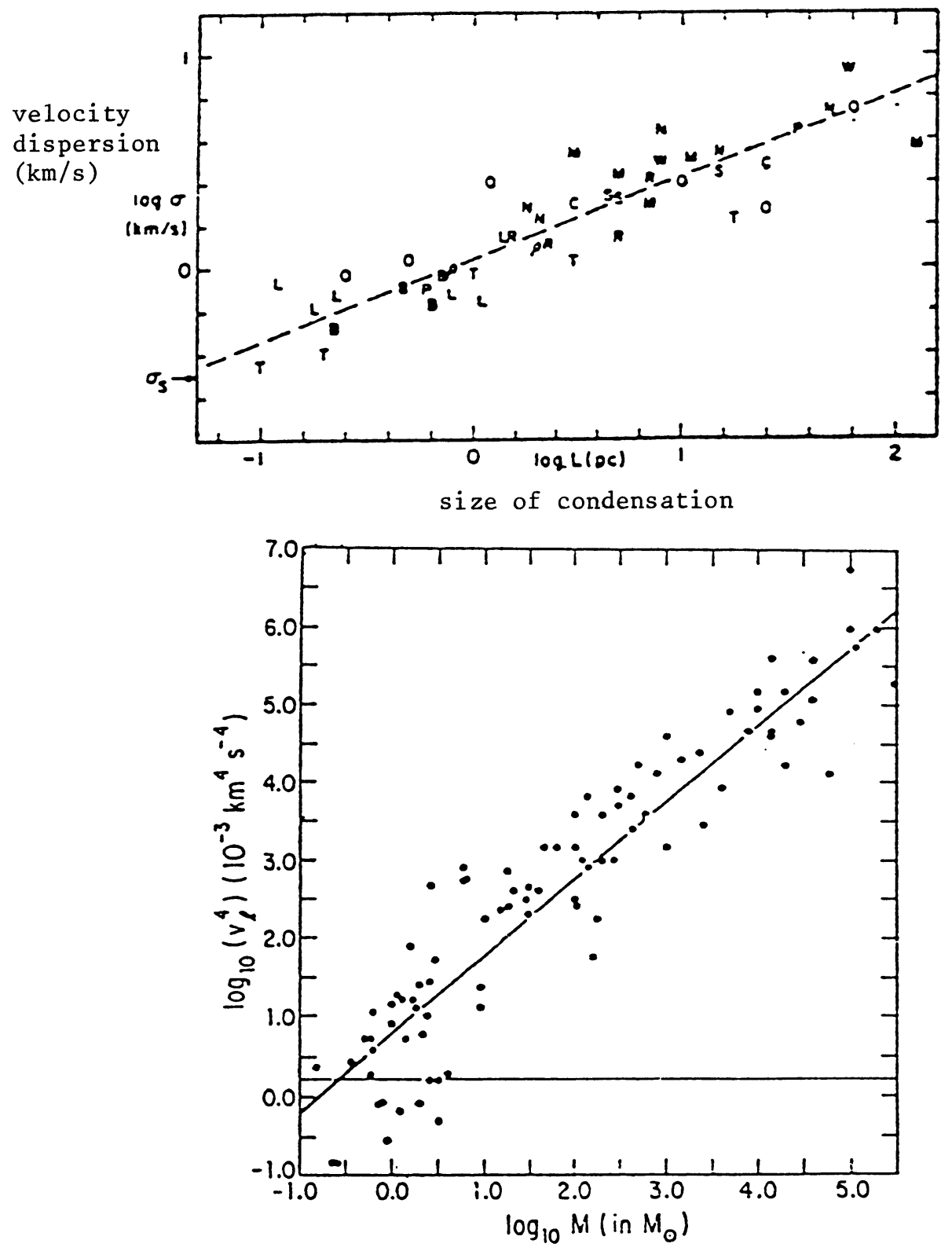

Fig. 3 (upper, from Myers 1985): log-log plot of three-dimensional velocity dispersion $\tau$ vs. size of region, showing that velocity dispersion varies approximately as $\mathrm{L}^{0.5}$.

(lower, from Henrickson and Turner 1984): log-log plot of internal velocity to the fourth power, on a scale $l$ vs. the mass of a cloud on that scale. The slope of the best-fit line is unity. 


\section{CORES}

In the traditional view of opacity-limited fragmentation, clouds could pass from the diffuse phase $\left(<100 \mathrm{~cm}^{-3}\right)$ through isothermal contraction until a density of $10^{12} \mathrm{~cm}^{-3}$ and thereafter continue contracting adiabatically. The predicted limit was a minimum Jeans' mass of $0.01 \mathrm{M}_{\odot}$. With modern data from molecular line astronomy, the situation has changed. The empirical data of Fig. 3 suggest that the minimum stellar mass will be of the order $0.1 \mathrm{M}_{\odot}$, and the fundamental units in the hierarchy of collapse are recognised to be "cores" observed in molecular clouds (Table 1). A new model by Falgarone and Puget (1985) for the stability of cores takes proper account of the ambient far-IR radiation observed in giant molecular clouds. In their mode1, the condensations have a core-halo structure, with the ambient far-IR field determining the core temperature, and the external UV radiation determining the extent of the halo and the total mass. The critical density is $10^{4} \mathrm{~cm}^{-3}$, above which the gas and the dust are thermally coupled, leading to the same structure for all models above this density. The temperature of the core is about 15-20 $\mathrm{K}$, even if there is no internal source of heating. There is a temperature minimum at the interface of the core and the halo. The predicted size of the core is typically 0.05 to $0.1 \mathrm{pc}$.

A possible example of such a structure, in the low-mass case, is that seen in rho $0 \mathrm{ph}$ in $2-\mathrm{cm} \mathrm{H} \mathrm{H}_{2} \mathrm{CO}$ emission. The VLA observations (Wadiak et al. 1985) indicate a mass of $1-4 \mathrm{M}_{\Theta}$, density $410^{6} \mathrm{~cm}^{-3}$ for a likely temperature of $20 \mathrm{~K}$. Examples of high mass cores may be the condensations seen in $\mathrm{M} 17$ in the radio continuum by Felli, Churchwell and Massi (1984). In their interpretation, the condensations on the VLA maps are neutral cores $>30 \mathrm{M}_{\odot}$, ionized on the outside only. They are otherwise distributed throughout the M17 molecular cloud, and only happen to be visible on radio continuum maps where they are "lit up" by the UV radiation from the ionizing stars of the M17 HII region.

In one recent interpretation (Turner 1984) the mass spectrum of cores has some fraction which are subsonic. These collapse to form low-mass stars. Once low-mass star formation has started, more gas is attracted into the region, to form more massive cores. Under the influence of an external agent (a shock induced by the accretion itself?, cloud-cloud collisions?), a massive core can collapse to form the first massive $s$ tar(s) in the region. These initial massive stars can then produce further compressions by their protostellar outflows, HII regions, main-sequence winds, and eventually by their supernovae. The process then runs away, producing more and more massive stars.

An alternative viewpoint (e.g. Elmegreen 1985), is that star formation is purely stochastic, with the spectrum of stellar masses simply corresponding to the probability spectrum of cloud fragments, that is, the observational fact that massive stars tend to be found associated with giant molecular clouds while low-mass clouds tend to have only low-mass stars, may just reflect the probability spectrum of fragmentation. For a Miller-Scalo initial mass function, one expects about 500 stars of typical mass $0.6 \mathrm{M}_{\odot}$ for every $\mathrm{OB}$ star 
of typical mass $23 \mathrm{M}_{\odot}$. Hence molecular clouds of only $10^{3} \mathrm{M}_{\odot}$ would be expected to use up nearly all their mass in producing the low-mass part of the initial mass function, and one would have to sample a few hundred such clouds to expect to find a single 0-type star.

Further arguments in favor of this stochastic approach to star formation are provided by Stahler (1985), who shows that the traditional dating of open clusters like NGC 2264 and NGC 6530 by isochrones fitted to the main sequence stars cannot be correct. The ages derived for different mass ranges correspond exactly to the pre-main-sequence contraction time, which cannot be correct, since the time scale is determined by physical processes unrelated to those acting when a star is on the main sequence. The reason why the traditional method is invalid is that the intrinsic dispersion in the main sequence part of the cluster $H-R$ diagrams is greater than the resolution provided by the theoretical isochrones in this area. Hence the observation by Herbig (1962) that massive stars form last in a cluster is not supported, according to Stahler, by the isochrone analyses. It is only true in a statistical sense, because the probability of formation of low mass stars is simply much higher than that of high-mass stars. Regardless of these two approaches, either that additional physics is required to produce high mass stars, after low-mass stars are formed, or the statistical interpretation, most authors agree that once high-mass stars form, they heat their surroundings, and raise the minimum Jeans mass. The dependence is estimated to be $M_{J} \sim \mathrm{T}^{2+\delta}$ (e.g. Silk 1977), where $\delta$ is positive and depends on grain properties. Formation of massive stars thus inhibits low mass star formation, and this concept is the basis for the model of Bi-modal star formation. These ideas were discussed by Mezger and Smith (1977) and have been put in rigorous form by Güsten and Mezger (1983). Observational evidence for bi-modality is summarized by Silk (1986). Larson (1986) explores the implications of the model of Bi-modal star formation in the context of a time-dependent initial mass function, decaying exponentially with a time constant of 4 Gyear. In the model of Güsten and Mezger, the massive stars form mainly (but not exclusively) in the spiral arms of the Galaxy, where they inhibit formation of stars less massive than $3 \mathrm{M}_{\odot}$ (Larson takes $2 \mathrm{M}_{\odot}$ as the lower limit). Stars of all masses $\left(0.1\right.$ to $\left.60 \mathrm{M}_{\odot}\right)$ form in the interarm regions.

The basic idea for the quantitative calculations of Güsten and Mezger is that the observed rate of production of Lyman continuum photons in a given region is directly proportional to the rate of formation of massive stars in that region.

$$
\mathrm{N}_{\text {Lyc }}=\mathrm{K} \psi_{\mathrm{OB}}
$$

where $\mathrm{N}_{\text {Lyc }}$ is the Lyman continuum production rate, $\psi_{O B}$ is the star formation rate for $O B$ stars, and $K$ is a constant

$$
\mathrm{K}=\langle\mathrm{N} / \tau \mathrm{M}\rangle \mathrm{OB}
$$

$\mathrm{K}=\langle\mathrm{N} / \tau \mathrm{M}>\mathrm{OB}$
where $\mathrm{N}, \tau$ and $\mathrm{M}$ the Lyman continuum rate, mean lifetime and mass for an average $O B$ star, where the weighting is taken over an initial mass function (IMF). For example, for the Miller-Scalo IMF,

$$
\mathrm{K}=3.210^{53} \frac{\text { photons } / \mathrm{sec}}{\mathrm{M}_{\odot} / \mathrm{yr}}
$$

In the model of Güsten and Mezger, the star formation rate for massive 
stars is

$$
\psi_{\mathrm{OB}}=\psi_{\mathrm{OB}}^{\mathrm{ia}}+\psi_{\mathrm{OB}}^{\mathrm{sa}}
$$

summed over the interarm (ia) and spiral arm (sa) regions. Furthermore, the star formation rate in the spiral arms, at galactic radius $R$, is assumed to be proportional to the amount of molecular gas passing through the spiral arms, namely

$$
\psi^{s a}=\nu \psi^{1 a}
$$

where $\nu$ is the difference between the rotational velocity $\Omega_{R}$ at galactic radius $R$ and the spiral pattern speed, $\Omega_{\mathrm{p}}$, normalised to unity in the neighbourhood of the sun, i.e.,

$$
\nu=\frac{\Omega_{\mathrm{R}}-\Omega}{\mathrm{p}}
$$

Hence, from (1), (3) and (4)

$$
\begin{aligned}
& \psi^{\mathrm{ia}}=\frac{\mathrm{N}_{\text {Lyc }}}{\mathrm{K}} \frac{1}{1+\nu} \\
& \psi_{\text {OB }}^{\text {sa }}=\frac{\mathrm{N}_{\text {Lyc }}}{\mathrm{K}} \frac{v}{1+v}
\end{aligned}
$$

Equations (5a) and ( $5 b)$ yield the star formation rates for massive stars (masses 10-60 $\mathrm{M}_{\odot}$ ) from derived values of $\mathrm{N}_{\text {Lyc }}$. Star formation rates for all other stellar mass ranges are obtained by simply scaling the values for $O B$ stars by the corresponding areas under the initial mass function. One may use three methods to derive the star formation rates:

Method I: discrete radio sources: In this method, the observed flux on the optically-thin part of the thermal radio continuum spectrum of HII regions is used to derive Lyman continuum photon rates. The distances of the HII regions are derived from the velocities of their radio recombination lines. Application of the (bi-modal) initial mass function gives the star formation rate for $O B$ stars in HII regions. A correction for the lifetime of HII regions (estimated from the ratio of numbers of field $O B$ stars to those in HII regions) gives the total star formation rate.

Method II: Integral approach: The deconvolution of extended thermal radio continuum flux, as a function of galactic longitude, gives the Lyman continuum rate as a function of galactic radius. Application of a bi-modal initial mass function then yields the star formation rate vs. galactic radius. In this second method, Güsten and Mezger have used current spiral arm models, to correct for the azimuthal asymmetry. Method III: Infrared Radiation: To derive the star formation rate, it is first necessary to decompose the infrared intensities from IRAS and from balloon observations into contributions from various components (Cox et al. 1986, Pajot et al. 1986 and Table 2). 
Table 2. Contributers to observed IR intensities (Cox et al. 1986)

\begin{tabular}{|c|c|c|}
\hline $\begin{array}{l}\text { Approx. } \\
\text { Wavelength } \\
\text { Range ( } \mu \mathrm{m})\end{array}$ & $\begin{array}{l}\text { Temp. of } \\
\text { emitters } \\
\quad(\mathrm{K})\end{array}$ & Type of emitters \\
\hline $\begin{array}{l}2-30 \\
5-30\end{array}$ & $\begin{array}{c}250-450 \\
--\end{array}$ & $\begin{array}{l}\text { grains in M-giant envelopes } \\
\text { small grains (Sellgren 1984); } \\
\text { polycyclic aromatic hydro- } \\
\text { carbons (Puget et al. 1985) }\end{array}$ \\
\hline $20-800$ & $30-40$ & dust heated by $O B$ stars \\
\hline $30-1000$ & $15-25$ & dust in HI clouds \\
\hline $50-1000$ & 14 & dust in molecular clouds \\
\hline
\end{tabular}

Cox et al. (1986) give simple analytic formulae to derive the Lyman continuum rate from the observed Far-IR flux of the warm $(30-40 \mathrm{~K})$ dust component.

These three methods yield satisfactory agreement on the star formation rate. The results (Fig. 4) show that while massive stars (mass range 10-60 $\mathrm{M}_{\odot}$, IMF-weighted typical mass $=23 \mathrm{M}_{\odot}$ ) form mainly in spiral arms, a significant fraction of massive stars is formed in the interarm region as well. For all stars (mass range 0.1 to $60 \mathrm{M}_{O}$, IMF-weighted typical mass $=0.6 \mathrm{M}_{\odot}$ ) the production rate is greater in the interarm region in the outer parts of the galaxy, but the interarm and spiral arm production rates are comparable for galactic radius $<5 \mathrm{kpc}$. Table 5 gives a global summary of the star formation rates, from the areas under the curves in Fig. 4.

Obviously the model of bi-modal star formation is only an approximation to what is happening in the Galaxy, as the initial mass function might vary from star cluster to cluster, and with galactic age as well. It is nevertheless encouraging that a relatively simple model explains a number of results. For example, since oxygen is produced only by massive stars, its abundance in the Galaxy should vary with the relative proportions of the spiral arm and interarm production rates. In fact the model for bi-modal star formation is in good agreement with the observed gradients of $160 / \mathrm{H}, \mathrm{He} / \mathrm{H},{ }^{12} \mathrm{C} /{ }^{13} \mathrm{C}$ and $18_{0} / 17_{0}$ as a function of galactic radius (Güsten and Mezger 1983, Güsten 1986). The model also removes the previous inconsistency between the derived star formation rates and the observed mass distribution in the galactic disk. The mass distribution of the Galaxy is determined by the component permanently locked up in stars (set mainly by the low-mass, interarm mode of star formation) and in remnants, while the recycling and enrichment of the interstellar matter is determined by the high-mass, spiral-arm mode of star formation.

Larson (1986) points out that the IMF presented by Scalo (1985) for nearby stars already has evidence for two peaks, and if the star formation rate declines on a time scale which is of the order of $4 \mathrm{Gyr}$, then the conclusion that the IMF is double-peaked becomes unavoidable. Larson's model includes a bi-modal IMF and a star formation rate which decreases exponentially with time. He concludes that the model of bi-modal star formation can account for the unseen mass in the solar 


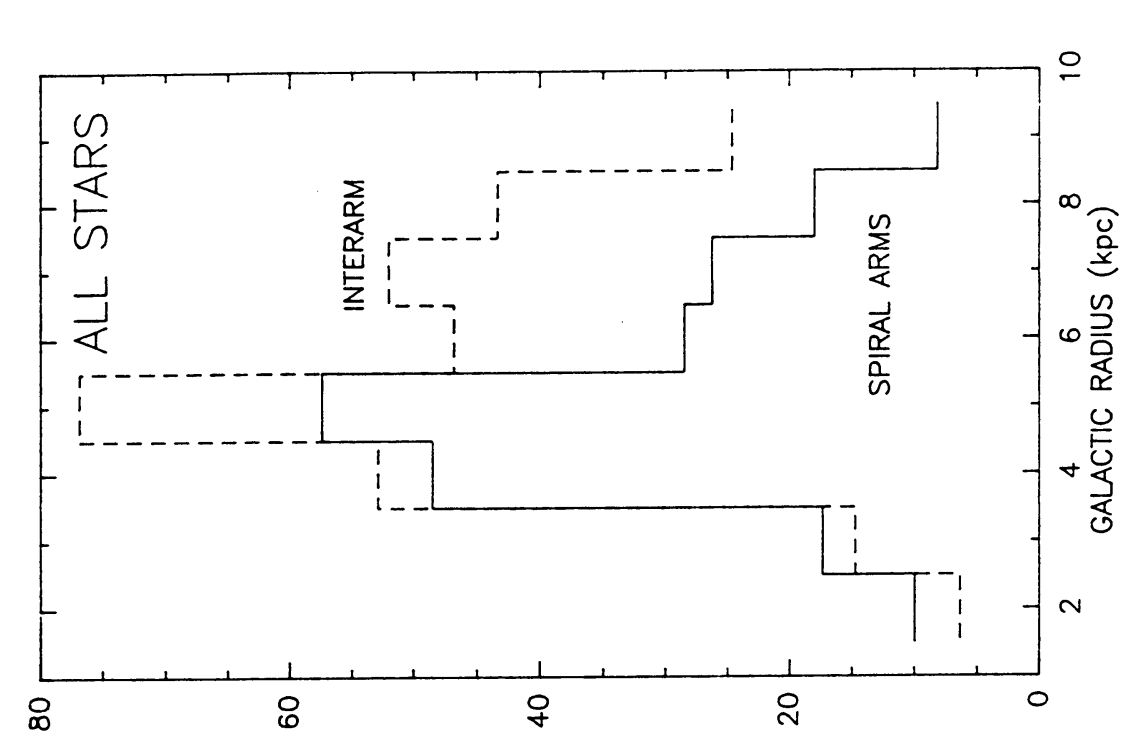

(

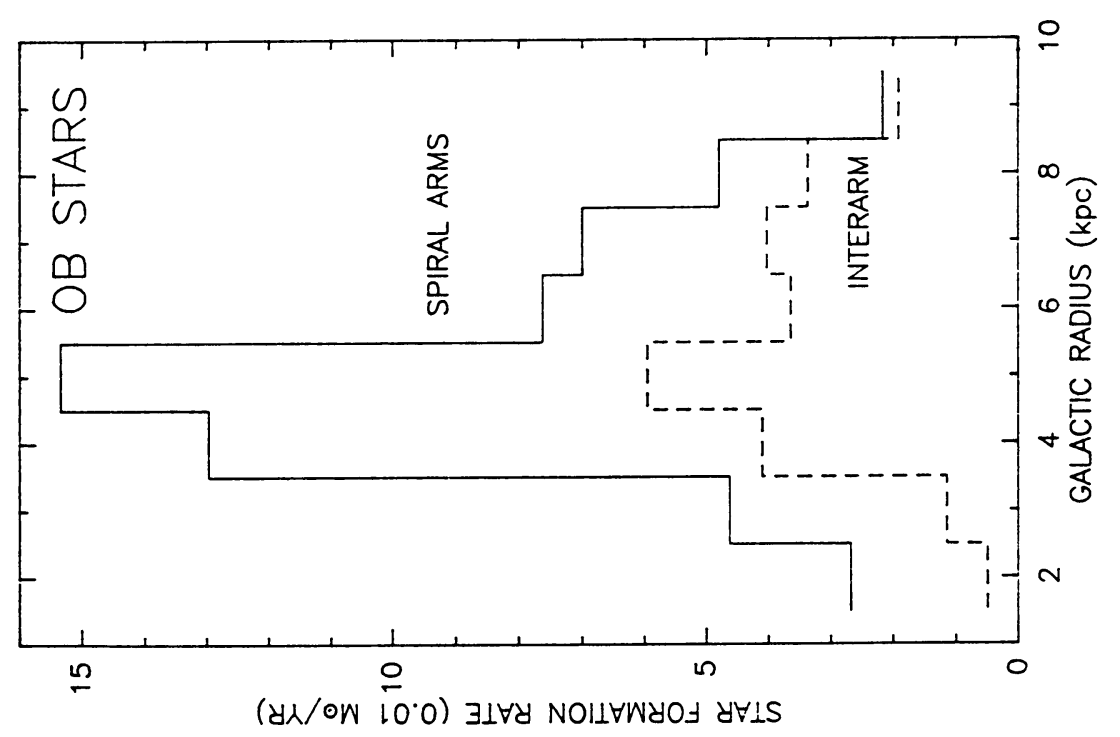

ง ลิ这

บัญ 需 $\infty$ 承 . $\rightarrow+\infty$ . نे 艾染 元 近望 $\hat{\mathrm{m}}$ (1) व \& 8 \%

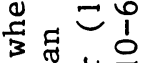
西岕こ 角穴 趈 古范 灵苗

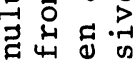

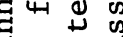
ส वृ: द क न ट ธี 氙

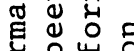
넝

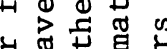
现 का का 4 क 山 씽. 计 \& 00 ๘ 必范 $+x$ on $\checkmark$ D 00 严 4 क $\rightarrow \pi$ 
Table 3: Star Formation Rates derived from the Bi-Modal Mode1 of Güsten and Mezger (1983) and a Miller-Scalo IMF

\begin{tabular}{llll}
\hline Category & $\begin{array}{l}\text { Region } \\
2<\mathrm{R}<10 \mathrm{kpc}\end{array}$ & $\begin{array}{l}\text { Range of } \\
\text { Stellar } \\
\text { Masses } \\
\left(\mathrm{M}_{\odot}\right)\end{array}$ & $\begin{array}{c}\text { Star formation } \\
\text { Rate }\end{array}$ \\
\hline OB stars & interarm & $10-60$ & 0.25 \\
OB stars & spiral arms & $10-60$ & 0.57 \\
OB stars & interarm + & $10-60$ & 0.82 \\
& spiral arm & & \\
al1 stars & interarm & $0.1-60$ & 3.2 \\
al1 stars & spiral arms & $3.0-60$ & 2.1 \\
al1 stars & interarm + & - & 5.3 \\
& spiral arm & & \\
\hline
\end{tabular}

neighbourhood (as remnants of massive stars), age-metallicity relations, without requiring gas infall into the galactic disk, and the colours of the bluest galaxies, as being dominated by the high-mass mode. A further inference is that young spiral galaxies must have been much more luminous than present-day ones, producing almost exclusively massive stars. Larson speculates that the dark matter in galactic halos may be the remnants of early generations of massive stars.

Figure 5 (from Turner 1984) gives a summary of some of the ideas reviewed here. In the context of $\mathrm{Bi}$-modal star formation, the left branch of Turner's diagram corresponds to the interarm mode, and to permanent lock-up of matter in low-mass stars, which determine the overall mass distribution in the Galaxy and thereby the galactic rotation curve. The right-hand side of the Figure corresponds to the formation of massive stars, and hence mainly to the spiral-arm component of the bi-modal model, which determines the re-cycling and metal enrichment of the interstellar medium. 


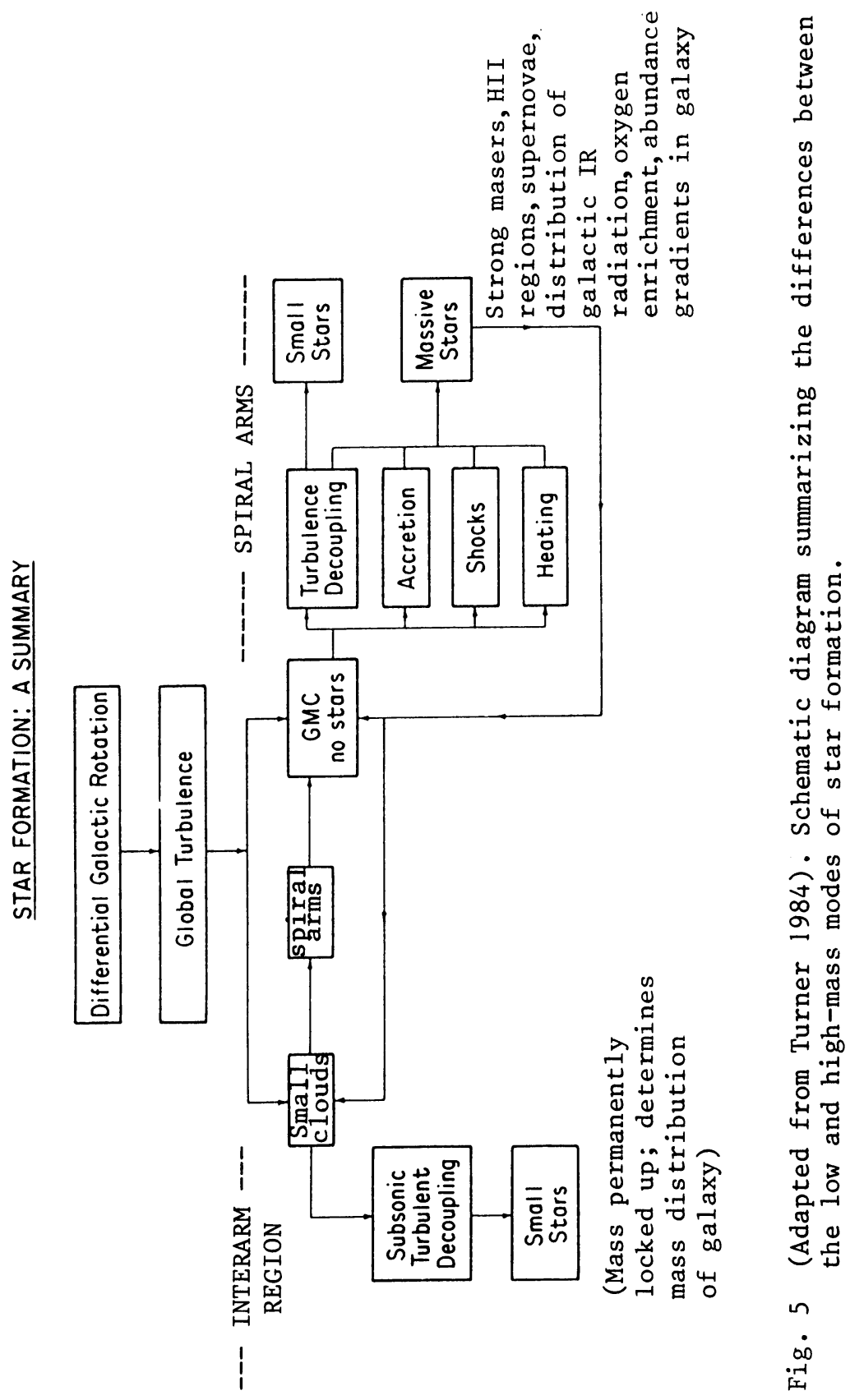




\section{REFERENCES}

Bash, F.N., Green, E., Peters, W.L. 1977, Ap.J. 217, 464

Beckwith, S., Natta, A., Salpeter, E.E., 1983, Ap.J. 267, 596

Cohen, R.S., Dame, T.M., Thaddeus, P. 1986, Ap.J. Supp 1.60, 695

Combes, F., Gérin, M. 1985, Astron.' Astrophys. 150, 327

Cox, P., Krüge1, E., Mezger, 1986, Astron. Astrophys . 155, 380

Elmegreen, B.G., 1985, in Naissance et Enfance des Etoiles, ed.

R. Lucas, A. Omont, R. Stora, North-Holland, Amsterdam, p. 257

Evans, N.J., 1985, in Protostars and Planets II, eds. D. Black and

M. Matthews, Tucson, Univ. Arizona Press, p. 175

Falgarone, E., Puget, J.L. 1985, Astron. Astrophys . 142, 157

Fel1i, M., Churchwe11, E.B., Massi, 1984, Astron. Astrophys. 136, 53

Fleck, R.C., 1980, Ap.J. 242, 1019; Ap.J. 246, L151; 1983 Ap.J. 272, L45

Güsten, R., Mezger, P.G. 1983, Vistas in Astronomy, 26, 159

Güsten, R., 1986, in Spectral Evolution of Galaxies, Advanced School of Astronomy, Erice, in press

Heiles, C., Stevens, M. 1986, Ap.J., 301, 331

Henrickson, R.N., Turner, B.E., 1984, Ap.J. 287, 200

Herbig, G.H., 1962, Ap.J. 135, 736

Jura, M., 1976, Astron. J., 81, 178

Kahn, F., 1974, Astron. Astrophys. 37, 149

Larson, R.B., 1978, Mon. Not. Roy. Astron. Soc. 184, 69

Larson, R.B., 1984, Mon. Not. Roy. Astron. Soc. 206, 197

Larson, R.B., 1985, Mon. Not. Roy. Astron. Soc. 214, 379

Larson, R.B., 1986, Mon. Noe. Roy. Astron. Soc. 218, 409

Leung, C.M., 1985, in Protostars and Planets II, eds. Black and

M. Matthews, Tucson, Univ. Arizona Press, p. 104

Mezger, P.G., 1986, in Luminous Stars and Associations in Galaxies,

IAU Symp. 116, eds. C. de Loore, A. Willis, P. Laskarides,

Reidel, Dordrecht, in press

Mezger, P.G., Smith, L.F., 1977, in Star Formation, IAU Symp. 75,

eds. T. de Jong, A. Maeder, Reidel, Dordrecht, p. 133

Mouschovias, T.Ch., 1976, Ap.J. 206, 753; 207, 141

Mouschovias, T.Ch., Morton, S.A., 1985, Ap.J. 298, 190; 298, 205

Myers, P., 1985, in Protostars and Planets II, eds. D. Black and

M. Matthews, Tucson, Univ. Arizona Press, p. 81

Myers, P.C., Dame, T.M., Thaddeus, P., Cohen, R.S., Silverberg, R.F.,

Dwek, E., Hauser, M.G., 1986, Ap.J. 301, 398

Nakano, T., 1984, Fund. Cosmic Phys., 9, 139

Nakano, T., Umebayashi, T., 1986, Mon. Not. Roy. Astron. Soc., 218, 663

Pajot, G., Boissé, P., Gispert, R., Lamarre, J.M., Puget, J.L.,

Serra, G., 1986, Astron. Astrophys., in press

Pudritz, R.E., Norman, C.A., 1983, Ap.J. 274, 677

Puget, J.L., Leger, A., Boulanger, F., 1985, Astron. Astrophys. 142, L19

Robinson, B.J., Manchester, R.N., Whiteoak, J.B., McCutcheon, W.H.,

1986, Astron. Astrophys., in prep.

Sanders, D.B., Clemens, D.P., Scoville, N.Z., Solomon, P.M.,

1986, Ap.J. Supp1. 60, 1

Scalo, J.M., 1985, Fund. Cosmic Phys., in press 
Schwartz, U.J., Troland, T.H., Albinson, J.S., Bregman, J.D., Goss, W.M., Heiles, C., 1986, Ap.J. 301, 320

Sellgren, K., 1984, Ap.J. 277, 623

Silk, J., 1977, Ap.J. 214, 154

Silk, J., 1986, in Luminous Stars and Associations in Galaxies, IAU Symp. 116, eds. C. de Loore, A. Willis, P. Laskarides, Reidel, Dordrecht, in press

Solomon, P., Sanders, D.B., 1985, in Protostars and Planets II, eds. D. Black and M. Matthews, Tucson, Univ. Arizona Press, p. 59

Stahler, S.W., 1985, Ap.J., 293, 207

Stark, A., Bally, J., 1987, this volume

Troland, T.H., Heiles, C., 1986, Ap.J. 301, 339

Turner, B.E., 1984, Vistas in Astronomy 27, 303

Uchida, Y., Shibata, K., 1985, Pub. Astron. Soc. Japan 37, 515

Wadiak, E.J., Wilson, T.L., Rood, R.T., Johnston, K.J., 1985, Ap.J. 295, L43

\section{DISCUSSION FOLLOWING REVIEW BY DOWNES}

KAHN: Cannot the support for the GMC's be provided by fast stellar winds from the newly formed stars? A fast wind (say $\sim 1000 \mathrm{~km} / \mathrm{s}$ ) will not cool once it is shocked. The resulting bubble of hot gas can provide support for a long time.

DOWNES: It does indeed seem plausible, as you show in your poster presentation at this symposium.

STROM: In NGC 2264 and in Orion, each region contains not only 0 and B stars, but stars as $1 \mathrm{c}$ in mass as $0.15 \mathrm{M}_{\Theta}$. In NGC 2264, the shape of the IMF, from $30 \mathrm{M}_{\odot}$ tu $0.15 \mathrm{M}_{\odot}$ is similar to the IMF inferred for the solar neighborhood. Hence, it seems difficult to defend pictures of bi-modal star formation which exclude formation of low-mass stars in regions of high-mass star formation in all cases.

DOWNES: The examples you cite would be considered by the authors of the bi-modal models to be cases of interarm star formation, in which the mass range of the IMF extends from 0.1 to $60 \mathrm{M}_{\Theta}$. Also, the IMFs of individual clusters may vary from place to place within the Galaxy, from the Galaxy to the Magellanic Clouds, and with galactic age. The model of bi-modal star formation should be understood as a global description, applying in a statistical sense to the Galaxy as a whole. Given that it is a simplified description of what is really happening, it is remarkable that the predictions of the bi-modal picture are so successful.

STROM: In NGC 2264, one can compare the rate of star formation as a function of time for low-mass stars only (i.e. stars which are all PMS stars). Among this group, Adams, Strom and Strom (1983) show that the lowest mass stars, in this sample, form first. 
DOWNES: Right. Noce that the critique of Stahler does not apply to the mass range in your sample $\left(0.2\right.$ to $\left.0.5 \mathrm{M}_{\odot}\right)$, but mainly to the mass range falling within the main-sequence band.

MOUSCHOVIAS: You said at the beginning of your talk that the consensus is that supersonic turbulence supports giant molecular clouds. I am not sure what constitutes a consensus in this case because, as a number of observers present at this meeting have been reporting, polarization observations (both optical and infrared) show orderly magnetic field structures in these clouds. If turbulence were so important, the fields would be completely tangled and no polarization would be seen.

DOWNES: On the contrary, the consensus is against the type of unreplenished supersonic turbulence of the type discussed in the literature twenty years ago. The magnetic field may have a large-scale ordered component as well as a smaller-scale, disordered component. The question is, which is the dominant support against gravity, the magnetic energy, or the kinetic energy of the disordered motions?

SHU: I would like to add to the point that Motschovias raised. It seems to me that one could accept all you say abou: bi-modal star formation without necessarily accepting the rest oi the "consensus" picture: In particular, I am puzzled by the claim that supersonic turbulence has anything to do with the formation of low-mass stars, since it seems to $f 1 y$ in the face of Myers' observation that the cores which have not yet formed (low-mass) stars have linewidths which are are 90 per cent thermal. How can the little residual "turbulence" in such cores have much to do with the collapse dynamics?

DOWNES: The argument for "turbulent" support probably applies on the larger scale lengths, that is, for the cloud complexes and for the envelopes of the cores. However, the low-mass cores may form only when this non-thermal support has largely dissipated. In the smaller-scale, higher-density regime of the cores, the support against gravity is provided by thermal pressure (as in the model by Falgarone and Puget), rather than by the residual bulk motions. 